\title{
Identifying the character of ferromagnetic Mn in epitaxial Fe/(Ga,Mn)As heterostructures
}

\author{
M. Sperl, ${ }^{1}$ F. Maccherozzi, ${ }^{2}$ F. Borgatti, ${ }^{3}$ A. Verna, ${ }^{4}$ G. Rossi, ${ }^{4,5}$ M. Soda, ${ }^{1}$ D. Schuh, ${ }^{1}$ G. Bayreuther,,${ }^{1}$ W. Wegscheider,${ }^{1}$ \\ J. C. Cezar, ${ }^{6}$ F. Yakhou, ${ }^{6}$ N. B. Brookes,${ }^{6}$ C. H. Back, ${ }^{1}$ and G. Panaccione ${ }^{4, *}$ \\ ${ }^{1}$ Institut für Experimentelle Physik, Universität Regensburg, D-93040 Regensburg, Germany \\ ${ }^{2}$ Soleil Synchrotron, L'Orme des Merisiers Saint-Aubin, BP 48, F-91192 Gif-sur-Yvette, France \\ ${ }^{3}$ ISMN-CNR, via Gobetti 101, Bologna, Italy \\ ${ }^{4}$ Laboratorio Nazionale TASC, INFM-CNR, in Area Science Park, S.S. 14, Km 163.5, I-34012 Trieste, Italy \\ ${ }^{5}$ Dipartimento di Fisica, Università di Modena e Reggio Emilia, Via A. Campi 231/A, I-41100 Modena, Italy \\ ${ }^{6}$ European Synchrotron Radiation Facility, BP 220, F-38043 Grenoble, France
}

(Received 18 November 2009; revised manuscript received 22 December 2009; published 27 January 2010)

\begin{abstract}
We demonstrate that the growth of $\mathrm{Fe} /(\mathrm{Ga}, \mathrm{Mn}) \mathrm{As}$ heterointerfaces can be efficiently controlled by epitaxy and that robust ferromagnetism of the interfacial $\mathrm{Mn}$ atoms is induced at room temperature by the proximity effect. X-ray magnetic circular dichroism and X-ray resonant reflectivity data, supported by theoretical calculations, were used to monitor both temperature and magnetic field dependence of the Mn magnetic moment in the semiconducting host. We identify distinct Mn populations, each of them with specific magnetic character.
\end{abstract}

DOI: 10.1103/PhysRevB.81.035211

PACS number(s): 75.50.Pp, 71.20.Nr, 78.70.Dm

\section{INTRODUCTION}

Diluted magnetic semiconductors (DMS) hold promises for integrating spin control in electronic devices. ${ }^{1,2}$ Although the correlation between magnetic and transport properties in DMS is known to be a crucial ingredient toward possible applications, the physical mechanisms governing both the magnetic character and the magnetization alignment of the metallic dopants in the semiconducting environment are open challenges of present DMS research. ${ }^{3-5}$ One further key subject arises from the Curie temperature, presently around $200 \mathrm{~K}$ in the most representative DMS material (Ga,Mn)As (Ref. 6) while ferromagnetic (FM) behavior well beyond room temperature (RT) would be required in future spintronics devices. A promising direction that goes beyond conventional methodologies is to tailor novel properties by exploiting interface effects in highly controlled heterostructures (HS), as extensively demonstrated in oxide-based materials. ${ }^{7-9}$ Following this approach, recent experimental efforts aimed at tuning specific magnetic properties in FM metal/DMS-based interfaces. In the case of direct exchangecoupled HS, the investigation of spin valve effect in MnAs/ $(\mathrm{Ga}, \mathrm{Mn}) \mathrm{As}$ at low temperature $(4.2 \mathrm{~K})$ found the apparent formation of a inhomogeneous magnetic spring in the $(\mathrm{Ga}, \mathrm{Mn}) \mathrm{As}$ region $^{10}$ and a decrease in exchange coupling after insertion of a spacer layer $\left[\mathrm{MnAs} / p\right.$-GaAs/(Ga,Mn)As]. ${ }^{11}$ Differently, HS with $3 d$ FM metals revealed (i) independent magnetization switching in $\left(\mathrm{Ni}_{80} \mathrm{Fe}_{20}\right) /(\mathrm{Ga}, \mathrm{Mn}) \mathrm{As}$, despite direct contact ${ }^{12}$ and (ii) FM behavior of $\mathrm{Mn}$ at $\mathrm{RT}$ at nonepitaxial $\mathrm{Fe} /(\mathrm{Ga}, \mathrm{Mn})$ As interfaces, with antiparallel alignment of the $\mathrm{Fe}$ and $\mathrm{Mn}$ moments. ${ }^{13}$ A firm understanding of the mechanisms involved in these effects requires on one side a full control of HS growth and on the other side the ability to probe the electronic and magnetic properties in a chemical and depth sensitive way. Here we report polarization-dependent $\mathrm{x}$-ray experiments on fully epitaxial $\mathrm{Fe} /(\mathrm{Ga}, \mathrm{Mn}) \mathrm{As} \mathrm{HS}$, where we are able to follow the field, temperature, and depth evolution of the different magnetic configurations of the Mn ions. Our results reveal the presence of a electronic configuration of the $\mathrm{Mn}$ ions under the proximity effect of the Fe overlayers, indicating that (a) Mn hybridization is playing a fundamental role in the magnetic properties of the system and (b) paramagnetic and ferromagnetic $\mathrm{Mn}$ (antiparallel to $\mathrm{Fe}$ ) have distinct spectroscopic fingerprints in an applied magnetic field, i.e., the different magnetic behavior in a sizeable part of the Mn atoms at the interface ( $\gtrsim 1 \mathrm{~nm})$ is "switched on" by the $\mathrm{Fe}$ overlayer.

\section{EXPERIMENTAL DETAILS}

The ferromagnetic (Ga,Mn)As samples (Mn 3-8 \% doping, $50-150 \mathrm{~nm}$ thick) have been grown by molecular-beam epitaxy (MBE). The rate for the low-temperature growth was about $0.6 \AA / \mathrm{s}\left(230^{\circ}-250^{\circ} \mathrm{C}\right)$. Subsequently the samples were transferred to a metal MBE for $\mathrm{Fe}$ evaporation. An optimized protocol to obtain contamination free and ordered surfaces was used, by combining low-temperature ultrahigh vacuum annealing $\left(150{ }^{\circ} \mathrm{C}\right)$ and soft $\mathrm{Ar}^{+}$sputtering $(500 \mathrm{eV}$, $45^{\circ}$ incidence angle) for a typical time of $30 \mathrm{~min}$. Details are given elsewhere. ${ }^{14,15}$ Reference samples were obtained by $\mathrm{HCl}$ etching. The Fe growth rate was controlled in situ by quartz monitors and the $\mathrm{Fe}$ overlayers were prepared as stepped wedges with discrete thickness values to ensure that the growth conditions were identical for all thicknesses in the covered range from 0 to $23 \mathrm{ML}$. The resulting well ordered surfaces were verified by reflective high-energy electron diffraction. Samples were covered by a 8-nm-thick epitaxial $\mathrm{Au}(001)$ layer to prevent oxidation. The magnetic anisotropy axis of both $\mathrm{Fe}$ and $(\mathrm{Ga}, \mathrm{Mn})$ As layer, determined by a superconducting quantum interference device magnetometer and by magneto-optical Kerr effect (MOKE), closely resembles $\mathrm{Fe} / \mathrm{GaAs}(001)$ and the nontreated (Ga,Mn)As/GaAs(001), respectively, excluding sizeable variations in the magnetic properties after the $\mathrm{Ar}^{+}$bombardment. ${ }^{14-16}$ Moreover, the Curie temperature of the sputtered $(\mathrm{Ga}, \mathrm{Mn})$ As is identical to the one of the as-grown samples $\left(T_{c}=67 \pm 3 \mathrm{~K}\right)$. Figure $1(\mathrm{a})$ presents MOKE results. At the wavelength used the Kerr 

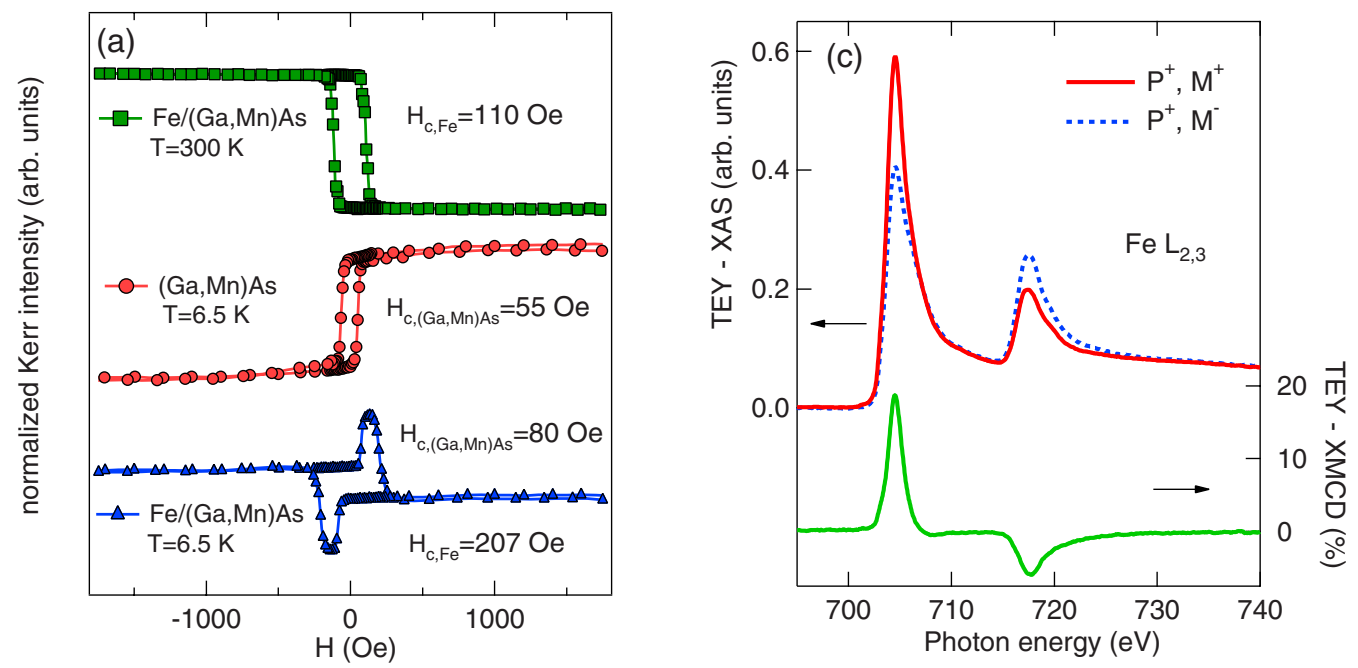

(b)
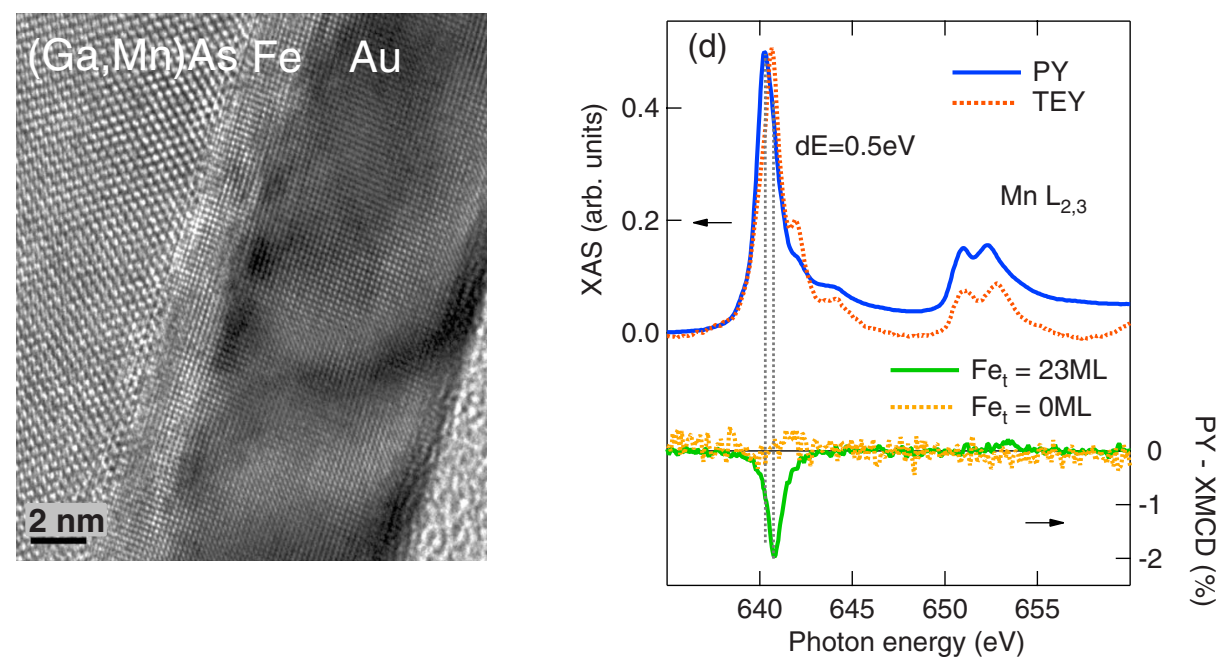

FIG. 1. (Color online) (a) Normalized MOKE data measured at 300 and $6.5 \mathrm{~K}$ for Fe/(Ga,Mn)As and (Ga,Mn)As. The Kerr contrast (measured with $\lambda=632 \mathrm{~nm}$ ) is opposite for $\mathrm{Fe}$ and $(\mathrm{Ga}, \mathrm{Mn}) \mathrm{As}$ which makes it possible to easily distinguish the two layers. The coercive field of Fe increases when going to lower temperatures as expected. A slight increase in $H_{c}$ is observed for the pure (Ga,Mn)As when Fe is deposited on top. (Panel b) High-resolution cross-sectional TEM image of the epitaxial Fe(2 nm)/(Ga,Mn)As(50 nm) sample, capped with 8 $\mathrm{nm}$ of $\mathrm{Au}$. The interface region is sharp and no intermixing is visible. The black horizontal line (bottom right) indicates the scale ( $2 \mathrm{~nm})$. (Panel c) Fe $L_{2,3}$ edge spectra as measured in TEY mode $(T=120 \mathrm{~K}, H=0.04 \mathrm{~T}, \mathrm{Fe}=23 \mathrm{ML})$, with circular polarization $\left(P^{+}\right)$and opposite magnetization $\left(M^{+}, M^{-}\right)$. Black arrows indicate the XAS (left) and XMCD (right) scale, respectively. (Panel d) Mn $L_{2,3}$ edge spectra as measured in TEY and PY mode $(T=120 \mathrm{~K}, H=0.04 \mathrm{~T}, \mathrm{Fe}=23 \mathrm{ML})$. Black arrows indicate the XAS (left) and XMCD (right) scale. Mn XAS spectra acquired in PY mode (blue/dark gray continuous line) and TEY mode (red/gray points) differ by 0.5 eV and display different fine structure at $L_{3}$ edge. XMCD signal at the Mn $L_{3}$ edge (green/light gray curve) is observed, indicating antiparallel magnetic coupling of Mn with respect to the Fe. No XMCD signal is measured in absence of the Fe overlayer (yellow/light gray curve in panel d, $\mathrm{Fe}=0 \mathrm{ML})$.

contrast for (Ga,Mn)As and Fe has opposite sign which makes it easy to separate the switching behavior of the two different layers. We show data at $300 \mathrm{~K}$ where only the $\mathrm{Fe}$ film produces a magnetic contrast and at $6.5 \mathrm{~K}$ for the $\mathrm{Fe} /$ GaMnAs film and for a pure (Ga,Mn)As film for comparison. We acquired x-ray absorption spectroscopy (XAS) and $\mathrm{X}$-ray circular magnetic dichroism (XMCD) spectra at the Mn and Fe $L$ edges at the ID08 dragon beamline (ESRF, France) and at the APE beamline of the Elettra Synchrotron (Trieste, Italy) in a temperature range of $10-300 \mathrm{~K}$ and a base vacuum $<5 \times 10^{-10}$ mbar. Total electron yield (TEY) and photon yield (PY, bulk sensitivity $>40 \mathrm{~nm}$ ) were acquired simultaneously. All spectra were normalized by the intensity of the incident beam. The TEY Fe XAS signal has been corrected for saturation effects, whereas PY mode has been preferred for $\mathrm{Mn}$, due to both the need of bulk sensitivity and to the dilution in GaAs, resulting in negligible self-absorption effects of the fluorescence signal. X-ray resonant magnetic scattering (XRMS) measurements have been carried out at ID08 in specular reflection geometry by fixing the incoming photon energy at the $L_{3} \mathrm{Mn}$ absorption peak and scanning the grazing angle $\theta$ from $0^{\circ}$ to $40^{\circ}$. Right circularly polarized radiation was used and reflectivity measurements were performed in remanence after having applied the magnetic field in opposite directions. Simulation and fitting of the x-ray resonant reflectivity measurements have 
been performed using the Pythonic Programming for Multilayer (PPM) software. ${ }^{17,18}$

\section{RESULTS AND DISCUSSION}

Figure 1 depicts the magnetic and structural characteristics of the $\mathrm{Fe} /(\mathrm{Ga}, \mathrm{Mn}) \mathrm{As} \mathrm{HS}$. The crystallinity of the $\mathrm{Au} / \mathrm{Fe} /$ (GaMn)As stack is confirmed by the transmission electron microscopy (TEM) image (panel b), revealing a sharp interface between $\mathrm{Fe}$ and $(\mathrm{Ga}, \mathrm{Mn}) \mathrm{As}$. Reference XAS-XMCD signals from the $\mathrm{Fe} L_{2,3}$ and $\mathrm{Mn} L_{2,3}$ edges are displayed in panels (c) and (d), for $23 \mathrm{ML} \mathrm{Fe}$ and $0.04 \mathrm{~T}$ at $120 \mathrm{~K}$, i.e., well above $T_{c}$ of the $(\mathrm{Ga}, \mathrm{Mn})$ As substrate. The PY spectrum is located at $\cong 0.5 \mathrm{eV}$ lower binding energy (BE) and it is less structured than the TEY one. The PY-XMCD displays FM behavior and antiparallel (AP) Mn orientation. ${ }^{13}$ We stress that, above $T_{c}$, a XMCD signal at $\mathrm{Mn} L_{2,3}$ edge is measured only in presence of the Fe overlayer. The yellow curve in panel (d) shows no XMCD at the Mn edge in the region free of $\mathrm{Fe}$, thus indicating a magnetic behavior switched on by the proximity effect of the Fe film.

From spectroscopic results, it is generally agreed that the two prevailing $\mathrm{Mn}$ electronic structures in $(\mathrm{Ga}, \mathrm{Mn}) \mathrm{As}$ are $\mathrm{Mn}_{\mathrm{Ga}}$ (Mn substitutional on the $\mathrm{Ga}$ site) and Mn forming oxides or aggregates near the surface, with a hybrid $d^{4}-d^{5}-d^{6}$ and a purely $d^{5}$ electronic configuration (localized Mn), respectively. The latter configuration has a more structured XAS spectrum, located at higher $\mathrm{BE}$ with respect to the $d^{4}-d^{5}-d^{6}$ one. ${ }^{19-22}$ A recent detailed study on the depth concentration of $\mathrm{Mn}$ in $(\mathrm{Ga}, \mathrm{Mn}) \mathrm{As}$ reveals the presence of $\mathrm{Mn}$ with $d^{5}$ configuration up to $6 \mathrm{~nm}$ from the interface, coexisting with $\mathrm{Mn}_{\mathrm{Ga}}$ and not related to oxide. ${ }^{23}$ Results in Fig. 1, obtained on controlled epitaxial systems with negligible oxide contribution indicate a surface-interface region with $d^{5}$-like character (TEY spectrum) and a bulk region, mainly representative of the $\mathrm{Mn}_{\mathrm{Ga}}$ (PY spectrum). For the sake of simplicity, we will refer in the following to $\mathrm{Mn}-1\left(\mathrm{Mn}_{\mathrm{Ga}}\right.$ substitutional), Mn-2 ( $d^{5}$-like Mn), and to Mn-AP (FM Mn antiparallel to $\mathrm{Fe}$ ). The evolution of the $\mathrm{Mn} L_{2,3} \mathrm{XMCD}$ signal vs applied magnetic field is presented in Fig. 2(a) $(T=120 \mathrm{~K}, \mathrm{Fe} 23 \mathrm{ML})$. At low field $(0.04 \mathrm{~T})$ only the Mn-AP dichroic component is visible at the $L_{3}$ edge, confirming previous results. ${ }^{13}$ Increasing the field, further components appear at lower BE, evolving in a structured line shape with a double peak at the $L_{2}$ edge and with the magnetization aligned parallel to the field. These components correspond to the paramagnetic contribution of Mn- 1 . The $\mathrm{XAS} / \mathrm{XMCD}$ arising from $\mathrm{Mn}-1$ has been associated to a mixed-valence ground state due to the large Mn $3 d$ hybridization with the surrounding $4 s p$ states. ${ }^{19,24,25}$ Numerical calculations indicated a mixed $80 \% d^{5}-20 \% d^{6} L$ ground state ( $L$ is a ligand hole). ${ }^{24,25}$ In Mn-AP, both the energy shift of the XMCD minimum and the line shape suggest a different valence state with lower occupancy of the $3 d$ levels, implying a lowering of the $d^{6}$ character and increment of the $d^{5}$ one.

In Fig. 2(b) the Mn-AP XMCD at $0.04 \mathrm{~T}$ is compared to atomic multiplet calculations, namely, a pure $\mathrm{Mn} d^{5}$ groundstate configuration based on the ligand field model, for an
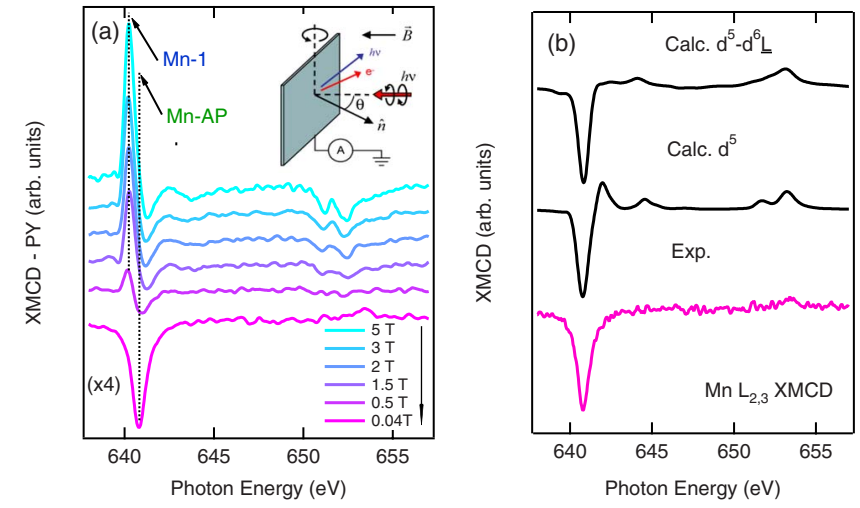

FIG. 2. (Color online) (Panel a) Evolution of the Mn XMCD line shape vs applied magnetic field $(T=120 \mathrm{~K}, \mathrm{Fe}=23 \mathrm{ML})$, measured in PY mode. The inset shows a sketch of the experimental geometry. (Panel b) Comparison of calculated and experimental (120 K, $0.04 \mathrm{~T}) \mathrm{Mn}-\mathrm{AP}$ XMCD. All curves have been normalized to the minimum of the experimental XMCD and are arbitrarily shifted in energy to align the theoretical to the experimental result. Features related to multiplet splitting effects are absent or smeared, as indicated by the vertical dashed lines. The interatomic screening and excessive electron-electron repulsion of the free ion were taken into account by reducing the Slater integrals to $80 \%$. The $2 p$ spin-orbit interaction was scaled to $103 \%$. An exchange field of $0.01 \mathrm{eV}$ was applied along the magnetization direction. The calculated spectra include a Lorentzian of $0.25(0.4) \mathrm{eV}$ for the $L_{3}\left(L_{2}\right)$ edge to account for intrinsic linewidth broadening and a Gaussian of $\sigma=0.1 \mathrm{eV}$ for instrumental broadening. For sake of comparison, all calculated spectra are normalized to the same amplitude and arbitrarily aligned in binding energy.

isolated ion in spherical symmetry,,$^{24,26,27}$ and a $d^{5}(50 \%)-d^{6}(50 \%)$ mixed-valence configuration. The $d^{5}(50 \%)-d^{6}(50 \%)$ mixed-valence XMCD has been calculated within the impurity Anderson model with the additional parameters: $E_{g}\left(d^{6}\right)=-3 \mathrm{eV}, E_{f}\left(d^{6}\right)=-4 \mathrm{eV}$, and $T_{t 2 g}=2 T_{e g}=2 . E_{g}$ and $E_{f}$ are related to the charge-transfer energies for ground and excited states while $T_{t 2 g}$ and $T_{e g}$ are the charge-transfer integrals for $t_{2 g}$ and $e_{g}$ orbitals, respectively. ${ }^{28}$ Introducing in the calculation further $d^{4}$ character or $d^{4}-d^{5}$ mixing without $d^{6}$ terms does not provide any better agreement to the experimental Mn XMCD. The comparison to the calculation highlights the smearing of the multiplet features in the experimental results and reveals unambiguously that Mn-AP XMCD does not bear a pure atomiclike character, suggesting a mixed-valence state with itinerant character. Moreover, the broadened features at the high-energy side of the $L_{3}$ XMCD that appear for a metalliclike condition are totally absent, thus excluding $\mathrm{Mn}$ alloying/clustering. The observed mixed-valence features suggest a rearrangement of the local density of states associated to changes in the local Mn environment that may be interpreted by the formation of an impurity band, similar to half-metallic systems, where Mn atoms have localized magnetic moments but delocalized $3 d$ electrons. ${ }^{29,30}$

To disentangle the Mn-AP ferromagnetic character from the paramagnetic one, we now need to assign the different contributions to the XMCD spectra. To this aim, a fitting procedure was performed, using a linear combination of ex- 

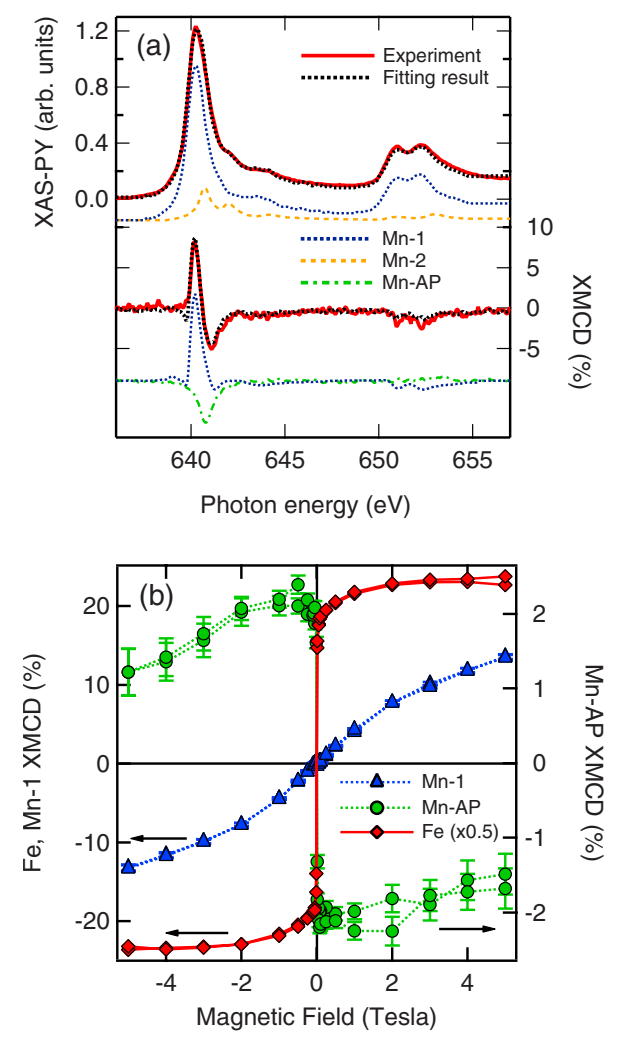

FIG. 3. (Color online) (a) Fitting results and experimental Mn $L_{2,3}$ XAS/XMCD spectra (black dashed curve and red/gray curve, respectively) using template spectra from Ref. 21 (Mn-1, blue/dark gray dashed curve and Mn-2 yellow/light gray dashed curve) and experimental Mn-AP XMCD (green/light gray dotdashed curve). Experimental curves correspond to $t(\mathrm{Fe})=23 \mathrm{ML}$, $T=120 \mathrm{~K}$, and $H=1 \mathrm{~T}$. Best agreement between experimental $\mathrm{XMCD}$ and fit is obtained by using only Mn-1 and Mn-AP component. (b) Hystereses cycles traced from the XMCD $L_{3}$ peak height at the $\mathrm{Mn}$ and $\mathrm{Fe}$ edges, in percentage of the XAS sum peak $\left(I_{+}-I_{-}\right) / 2$, for $T=120 \mathrm{~K}, \mathrm{Fe}=23 \mathrm{ML}$. Black arrows indicate the $\mathrm{Fe}$ XMCD scale (left) and Mn XMCD one (right). The Mn-1 (blue/ dark gray triangles) and Mn-AP (green/light gray circles) hystereses were extracted by fitting the $L_{3}$ XMCD spectra at each magnetic field, using the same fitting procedure of panel (a) (linear combination of Mn-1 and Mn-AP components). The resulting Mn-1 behavior is purely paramagnetic, as expected above $T_{c}$ of $(\mathrm{Ga}, \mathrm{Mn}) \mathrm{As}$. Experimental values of Fe XMCD (red/gray diamonds) are multiplied by 0.5 for sake of comparison.

perimental Mn-1,2 XAS and XMCD spectra from Ref. 22, constrained to reproduce XAS and XMCD data vs magnetic field, temperature, and Fe thickness. The Mn-AP XMCD cannot be reproduced by the superposition of $\mathrm{Mn}-1$ and $\mathrm{Mn}-2$, and has to be explicitly included in the fitting routine. Results for $120 \mathrm{~K}, 1 \mathrm{~T}$, and $23 \mathrm{ML}$ of Fe are presented in Fig. 3(a). We find only two contributions in the XMCD spectra: paramagnetic from bulk Mn-1 $\left(T>T_{c}\right)$ and ferromagnetic from Mn-AP. Although a $1 \mathrm{~T}$ field is applied, no paramagnetic contribution associated to $\mathrm{Mn}-2$ character is found, thus confirming the absence of Mn oxide in the epitaxial HS. Disentangling the different Mn character in XMCD spectra allows tracing the evolution vs magnetic field, i.e., to reconstruct site selective (Mn-1 and Mn-AP) hysteresis loops, as
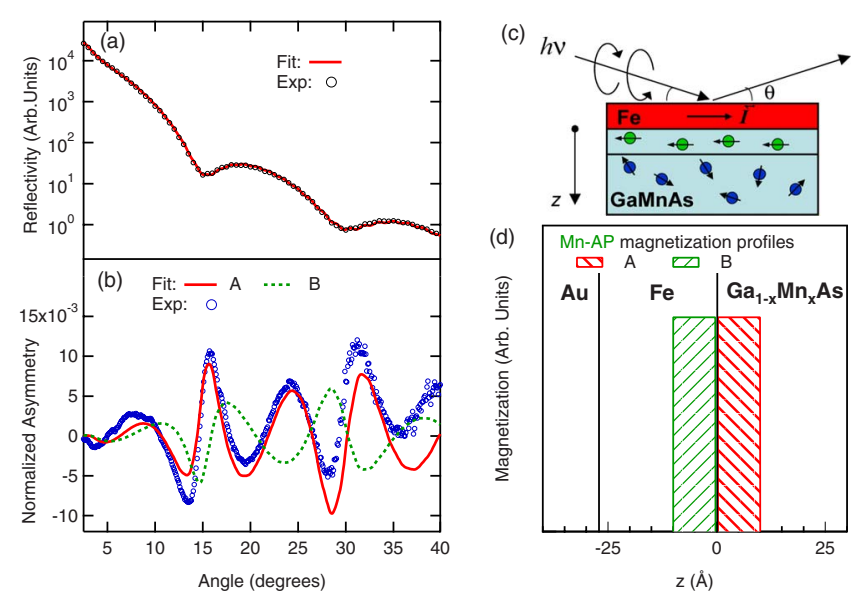

FIG. 4. (Color online) (a) Specular x-ray reflectivity at $138 \mathrm{~K}$ collected with a photon energy of $640.4 \mathrm{eV}$ (black circles) compared with fitting results obtained with the PPM program (red/gray line). Values used in the fitting are (thickness and roughness in $\AA$, respectively) (GaMn)As, 369 and 3.0. Fe 27 and 4.1. Au 41 and 2.1. GaAs substrate with roughness $1.0 \AA$. (b) Experimental asymmetry ratio AR (blue/dark gray circles), as defined in the text. Comparison of the experimental AR with two simulated magnetic profiles, i.e., a 10 - $\AA$-thick magnetic layer of Mn ions located in the $(\mathrm{Ga}, \mathrm{Mn}) \mathrm{As}$ environment (red/gray curve, panel d) or in the Fe film (green/light gray curve, panel d). (c) Sketch of the experimental geometry used for XRMS experiments.

presented in Fig. 3(b). The Mn-AP hysteresis matches the Fe one up to $2 \mathrm{~T}$, indicating the robustness of the magnetic coupling. At higher magnetic fields the observed partial reduction in the Mn magnetic moment $(\cong 40 \%)$ suggests a reorientation of the Mn magnetization in a direction parallel to the field. Having identified the novel magnetic and electronic behavior of Mn-AP, we now address the distribution profile of Mn-AP across the interface, by means of XRMS results. Figure 4(a) reports the $\theta-2 \theta$ scan obtained at $h \nu=640.4 \mathrm{eV}$ and $T=138 \mathrm{~K}$, averaging the reflectivity curves for parallel and antiparallel relative orientation of photon helicity and magnetic field. Such a signal is insensitive to the magnetic moments of the sample and depends only upon the electron charge distribution and chemical properties. In Fig. 4(b) we report the asymmetry ratio $\mathrm{AR}=(r \uparrow \uparrow-r \uparrow \downarrow) /(r \uparrow \uparrow-r \uparrow \downarrow)$, where $(r \uparrow \uparrow)$ and $(r \uparrow \downarrow)$ are the reflectivity values measured for parallel and antiparallel orientation of the photon helicity and magnetization, respectively. The AR value gives information about the distribution of the Mn magnetic moment in the sample. We fitted the $\theta-2 \theta$ scan using the thickness and roughness of the various layers $[\mathrm{Au}, \mathrm{Fe},(\mathrm{GaMn}) \mathrm{As}, \mathrm{GaAs}]$ as fitting parameters. Absorption coefficients for the Mn species have been obtained experimentally from XAS measurements. The real part of the refractive index is obtained from the imaginary part, which is directly proportional to the absorption coefficient, through Kramers-Kroenig transformation. The fitting results well reproduce the experimental curve (the values of the best fit parameters are listed in the caption of Fig. 4), in good agreement with TEM results. Considering a distribution of $\mathrm{Mn}$ atoms antiparallely oriented with respect to $\mathrm{Fe}$, we are able to reproduce maxima and minima of the measured oscillations: best agreement is found 
using a 10 - $\AA$-thick layer of Mn atoms with a constant steplike magnetic profile in $(\mathrm{Ga}, \mathrm{Mn}) \mathrm{As}$. We also simulated the behavior of the same Mn thickness, but with Mn diffused into the Fe layer, in antiparallel configuration. The period of oscillations is almost equal but the positions of the maxima and minima are inverted. This excludes a sizeable presence of magnetic $\mathrm{Mn}$ atoms into the Fe film. We stress that the simple steplike magnetization profile used in the simulation, although able to reproduce the XRMS data, should be considered as a lower estimate of the Mn-AP magnetic depth profile.

\section{CONCLUSIONS}

In conclusion, by the use of a broad range of chemical sensitive spectroscopic tools we have provided a complete description of the Mn magnetic properties in fully epitaxial Fe/GaMnAs HS. The use of the magnetic proximity effect between $\mathrm{Fe}$ and $\mathrm{Mn}$ made it possible to reveal a novel electronic configuration of the interfacial Mn atoms, with different magnetic behavior with respect to the bulk ones. Our original approach demonstrates that important insights in the fundamental interactions driving ferromagnetism in DMSbased systems can only be obtained by tailoring novel properties in controlled heterostructures. Future efforts in the direction of hybrid metal/DMS ferromagnetic structures may achieve the control of switching fields and Curie temperature, opening a completely new avenue for the design of hybrid metal/DMS/semiconductor structures for future spintronic devices.

\section{ACKNOWLEDGMENTS}

Financial support by the DFG through the SFB 689 is gratefully acknowledged. This work has been partially funded by CNR-INFM. Thanks are due to Alessandro Mirone for fruitful discussion and support for the use of the PPM code, and to Stefano Nannarone and Bruce A. Davidson for fruitful discussions on XRMS technique. A.V. was supported by the FVG Regional Project SPINOX funded by Legge Regionale 26/2005 and the decreto 2007/LAVFOR/ 1461 . *panaccioneg@elettra.trieste.it

${ }^{1}$ S. A. Wolf, D. D. Awschalom, R. A. Buhrman, J. M. Daughton, S. von Molnár, M. L. Roukes, A. Y. Chtchelkanova, and D. M. Treger, Science 294, 1488 (2001).

${ }^{2}$ D. D. Awschalom and M. E. Flatté, Nat. Phys. 3, 153 (2007).

${ }^{3}$ A. H. Macdonald, P. Schiffer, and N. Samarth, Nature Mater. 4, 195 (2005)

${ }^{4}$ I. Zutic, J. Fabian, and S. Das Sarma, Rev. Mod. Phys. 76, 323 (2004).

${ }^{5}$ G. Wastlbauer and J. A. C. Bland, Adv. Phys. 54, 137 (2005), and references therein.

${ }^{6}$ K. Olejnik, M. H. S. Owen, V. Novák, J. Mašek, A. C. Irvine, J. Wunderlich, and T. Jungwirth, Phys. Rev. B 78, 054403 (2008); J. Cryst. Growth 311, 2151 (2009).

${ }^{7}$ J. Chakhalian, W. Freeland, H.-U. Habermeier, G. Cristiani, G. Khaliullin, M. van Veenendaal, and B. Keimer, Science 318, 1114 (2007).

${ }^{8}$ H. Yamada, Y. Ogawa, Y. Ishii, H. Sato, M. Kawasaki, H. Akoh, and Y. Tokura, Science 305, 646 (2004).

${ }^{9}$ C. Chappert, A. Fert, and F. Nguyen Van Dau, Nature Mater. 6, 813 (2007).

${ }^{10}$ M. Zhu, M. J. Wilson, B. L. Sheu, P. Mitra, P. Schiffer, and N. Samarth, Appl. Phys. Lett. 91, 192503 (2007).

${ }^{11}$ M. Zhu, M. J. Wilson, P. Mitra, P. Schiffer, and N. Samarth, Phys. Rev. B 78, 195307 (2008).

${ }^{12}$ S. Mark, C. Gould, K. Pappert, J. Wenisch, K. Brunner, G. Schmidt, and L. W. Molenkamp, Phys. Rev. Lett. 103, 017204 (2009).

${ }^{13}$ F. Maccherozzi, M. Sperl, G. Panaccione, J. Minár, S. Polesya, H. Ebert, U. Wurstbauer, M. Hochstrasser, G. Rossi, G. Woltersdorf, W. Wegscheider, and C. H. Back, Phys. Rev. Lett. 101, 267201 (2008).

${ }^{14}$ F. Maccherozzi, G. Panaccione, G. Rossi, M. Hochstrasser, M. Sperl, M. Reinwald, G. Woltersdorf, W. Wegscheider, and C. H.
Back, Surf. Sci. 601, 4283 (2007).

${ }^{15}$ F. Maccherozzi, G. Panaccione, G. Rossi, M. Hochstrasser, M. Sperl, M. Reinwald, G. Woltersdorf, W. Wegscheider, and C. H. Back, Phys. Rev. B 74, 104421 (2006).

${ }^{16} \mathrm{Fe}$ growth on etched substrates results in a polycrystalline morphology, with high degree of magnetic disorder. In this case, no detectable XMCD at the Mn edge has been found.

${ }^{17}$ L. G. Parratt, Phys. Rev. 95, 359 (1954) PPM code is available at http://www.esrf.eu/computing/scientific/PPM/ppm.html

${ }^{18}$ B. L. Henke, E. M. Gullikson, and J. C. Davis, At. Data Nucl. Data Tables 54, 181 (1993).

${ }^{19}$ K. W. Edmonds, P. Bogusławski, K. Y. Wang, R. P. Campion, S. N. Novikov, N. R. S. Farley, B. L. Gallagher, C. T. Foxon, M. Sawicki, T. Dietl, M. Buongiorno Nardelli, and J. Bernholc, Phys. Rev. Lett. 92, 037201 (2004).

${ }^{20}$ K. W. Edmonds, W. Edmonds, N. R. S. Farley, R. P. Campion, C. T. Foxon, B. L. Gallagher, T. K. Johal, G. van der Laan, M. MacKenzie, J. N. Chapman, and E. Arenholz, Appl. Phys. Lett. 84, 4065 (2004).

${ }^{21}$ A. A. Freeman, K. W. Edmonds, N. R. S. Farley, S. V. Novikov, R. P. Campion, C. T. Foxon, B. L. Gallagher, E. Sarigiannidou, and G. van der Laan, Phys. Rev. B 76, 081201(R) (2007).

${ }^{22}$ K. W. Edmonds, G. van der Laan, A. Freeman, N. R. S. Farley, T. K. Johal, R. P. Campion, C. T. Foxon, B. L. Gallagher, and E. Arenholz, Phys. Rev. Lett. 96, 117207 (2006).

${ }^{23}$ F. Kronast, R. Ovsyannikov, A. Vollmer, H. A. Dürr, W. Eberhardt, P. Imperia, D. Schmitz, G. M. Schott, C. Ruester, C. Gould, G. Schmidt, K. Brunner, M. Sawicki, and L. W. Molenkamp, Phys. Rev. B 74, 235213 (2006).

${ }^{24}$ G. van der Laan and B. T. Thole, Phys. Rev. B 43, 13401 (1991).

${ }^{25}$ H. Ohldag, V. Solinus, F. U. Hillebrecht, J. B. Goedkoop, M. Finazzi, F. Matsukura, and H. Ohno, Appl. Phys. Lett. 76, 2928 (2000).

${ }^{26}$ T. Cowan, The Theory of Atomic Structure and Spectra (Univer- 
sity of California Press, Berkeley, CA, 1981).

${ }^{27}$ Systematic investigation of crystal-field effects that could lead to such XMCD curve did not produce better agreement with the experimental XMCD. Therefore the theoretical XMCD for a pure $\mathrm{Mn} d^{5}$ ground state in Fig. 2 was obtained in $\mathrm{O}(3)$ spherical symmetry.

${ }^{28}$ F. M. F. de Groot, Coord. Chem. Rev. 249, 31 (2005).
${ }^{29}$ J. Kübler, A. R. Williams, and C. B. Sommers, Phys. Rev. B 28 , 1745 (1983).

${ }^{30}$ N. D. Telling, P. S. Keatley, G. van der Laan, R. J. Hicken, E. Arenholz, Y. Sakuraba, M. Oogane, Y. Ando, K. Takanashi, A. Sakuma, and T. Miyazaki, Phys. Rev. B 78, 184438 (2008). 